\section{Flow Filling a Curved Pipe}

A small scale experiment was designed to study the propagation of the front of a viscous fluid filling a curved pipe. Several Newtonian fluids with different viscosities and a non-Newtonian fluid have been used. The experiments show that there exists a minimum speed for completely filling the pipe, which depends on the parameters of the experiment (di-ameter $\mathrm{d}$ and radius of curvature $\mathrm{R}$ of the pipe, kinematic viscosity $v$ of the fluid). Appropriate dimensionless numbers are introduced to characterize the flow and optimal filling conditions.

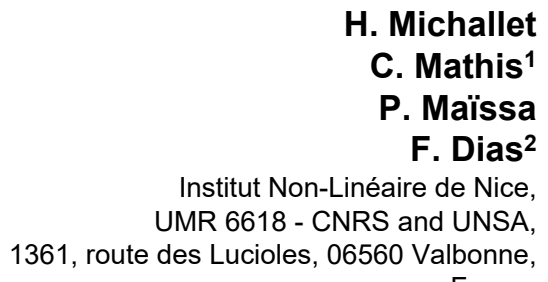

France

\section{Introduction}

Prestressed concrete is commonly used in construction. To prevent the prestressed strands from any corrosion, a cement solution is generally injected in pipes of complex geometry. The pipe has to be completely filled in order to avoid weak zones in the structure. The aim of the present study is to observe experimentally the propagation of the front of a viscous fluid injected in a curved pipe, in order to understand the reasons for the appearance of unfilled zones. The solution used in construction is composed of cement, water, liquefier, and retarder. It is a non-Newtonian fluid with air bubbles and particles in suspension. As a first step, we mainly focus in this paper on the filling by Newtonian fluids.

Permanent flows in pipes have been widely studied [1], particularly in the case of curved pipes [2-4] and in engineering applications $[5,6]$. However, pipe flows involving a propagating front or interface remain poorly understood. The problem of an airwater interface propagating in straight inclined pipes has been addressed only recently $[7,8]$. As stated above, in industrial applications such as construction, the fluid is often mixed with air bubbles and the flow ought to be treated as a slug flow (see for example $[9,10]$ for recent work on slug flows in pipes). But the emphasis of the present work is in the propagation of the interface which clearly separates the gas from the liquid.

The front propagation of a perfect fluid in a horizontal pipe was studied by Benjamin [11]. See also the review by Simpson [12] on gravity currents for related studies and Asavenant and VandenBroeck [13]. Benjamin analyzed the front in terms of a "cavity flow" displacing a fluid beneath it. The two-dimensional geometry is shown in Fig. 1(a) (Benjamin also considered the case of a circular cross-section). Note that Benjamin studied only the case where the free surface detaches with an angle of 60 degrees. By applying conservation of mass, momentum, and energy, he found that there is a unique solution, characterized by

$$
\frac{U}{\sqrt{g d}}=\frac{1}{2}, \frac{u}{\sqrt{g h}}=\sqrt{2}, \frac{h}{d}=\frac{1}{2},
$$

where the meaning of the various symbols is shown in Fig. 1. In other words, the cavity fills half of the box. An experiment was suggested by Benjamin to realize closely this flow. Liquid initially fills a long rectangular box closed at both ends and fixed horizontally. One end is then opened, and under the action of gravity the liquid flows out freely from this end. It can be expected that, after

${ }^{1}$ Permanent address: Laboratoire des Ecoulements Géophysiques et Industriels (UJF-INPG-CNRS), BP53, 38041 Grenoble Cedex 9, Fax: +33 4768252 71; E-mail: herve.michallet@hmg.inpg.fr.

${ }^{2}$ Corresponding author, present address: Ecole Normale Supérieure de Cachan CNRS, CMLA UMR8536, 61 avenue du Président Wilson, 94235 Cachan Cedex; Email: dias@cmla.ens-cachan.fr. the transient effects of starting have disappeared, the air-filled cavity replacing the volume of the ejected fluid will progress steadily along the box. Observed in a frame of reference travelling with the front of the cavity, the motion of the liquid will appear to be steady, as shown in Fig. 1(a). If the effects of viscosity and surface tension are neglected, the velocity of the cavity relative to a stationary observer will be $U$, and, since $h=d / 2$, the liquid will discharge from the open end with the same velocity. In addition to the solution studied by Benjamin, there is also a one-parameter family of solutions in which the free surface detaches from the box tangentially [14]. Such a solution is plotted in Fig. 1(b). At the exit of the box, the Froude number $u(g h)^{-1 / 2}$ for these solutions is between 1 and $\sqrt{2}$. The limit $\sqrt{2}$ corresponds to Benjamin's solution, while the limit 1 corresponds to the vanishing of the cavity. Using conservation of mass, momentum and energy, one finds the relation

$$
\frac{u}{\sqrt{g h}}=\sqrt{\frac{d}{h}} .
$$

Unfortunately, simple arguments based on conservation of mass, momentum and energy do not give as much information in the case of a curved channel.

It is nevertheless important to keep in mind that the Froude number prevails to determine the size of the cavity and thus the ability to fill the pipe. This point will be discussed in the section where we consider various dimensionless numbers. Before that, we first describe the manner in which the experiments have been conducted. Then we present and discuss the experimental results.

\section{Experimental Setup}

The experimental setup is shown in Fig. 2. Gravity acts downwards. A transparent PVC pipe ("Tubclair') of inner diameter $d$ is curved with a radius $R$. Different diameters and radii were considered: $0.6 \leqslant d \leqslant 2 \mathrm{~cm} ; 5 \leqslant R \leqslant 30 \mathrm{~cm}$. The special case of a horizontal straight pipe was also investigated. The fluid was injected with a centrifugal pump at a constant mean flow velocity (noted $U$ ).

Several fluids were considered in order to investigate the effects of density $\rho$, surface tension $\sigma$, and mainly kinematic viscosity $\nu$ (see Table 1). A series of runs has been performed with a nonNewtonian fluid (gel). Its viscosity has been measured with a plane-plane viscosimeter (see Fig. 3).

We assume that capillary effects are negligible, so that $d \gg L_{c}$ where $L_{c}=\sigma^{1 / 2}(\rho g)^{-1 / 2}$ is the capillary length $\left(\sigma=73 \mathrm{gs}^{-2}\right.$ for water and $\sigma=21 \mathrm{gs}^{-2}$ for silicone). In the case of water $L_{c}$ $=0.26 \mathrm{~cm}$ and this assumption might be a shortcoming for small diameters. We nevertheless performed a few runs with water contaminated with wetting agents in order to diminish $L_{c}$ : the behavior and the results were the same within measurements errors. Moreover, the results presented in this paper are limited to $d \geqslant 1$ $\mathrm{cm}$. 
(a)

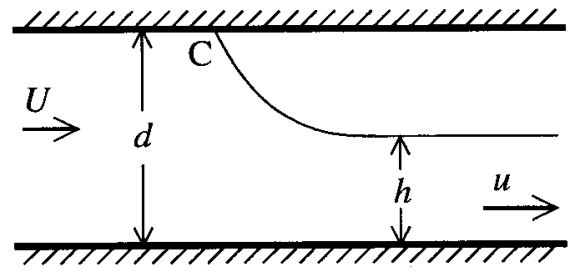

(b)

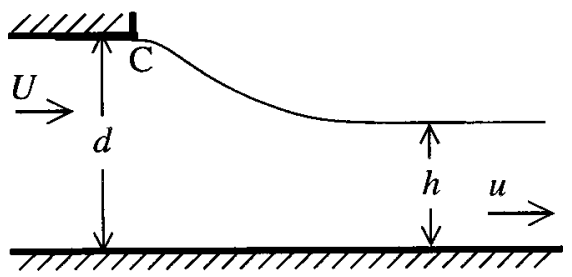

Fig. 1 Steady two-dimensional flow past a cavity. (a) Solution with the free surface leaving the wall with a 60 degree angle. (b) Solution with the free surface leaving the wall tangentially. The point $C$ denotes the detachment point.

\section{Observations}

For a very low flow rate (a typical mean flow velocity is $U$ $\sim 0.1 \mathrm{~cm} / \mathrm{s}$ ) and a sufficiently large pipe section (so that $d \gg L_{c}$ ) the interface is more or less horizontal in the upflow part of the pipe. The flow is dominated by gravity. In the downflow part of the pipe the liquid is creeping down the pipe.

If $U$ is slightly increased, the interface becomes more or less perpendicular to the pipe when rising. At the top, the interface deforms as shown in Fig. 2. The intersection between the interface and the upper part of the pipe (point $\mathrm{C}$ in Fig. 2) is a stagnation point.

If $U$ is increased, the point $\mathrm{C}$ moves (interface in dotted line in Fig. 2). As soon as $U$ is decreased back to zero, the pipe is emptying and the interface moves back to the top.

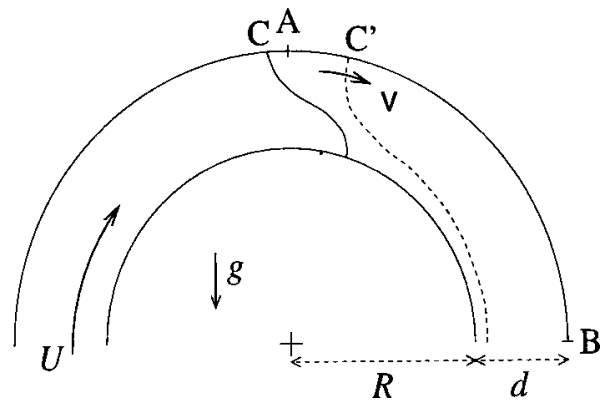

Fig. 2 Diagram of the flow and notation. The interface is shown at two different times in solid and dotted lines.

Table 1 Fluids used in the experiments.

\begin{tabular}{|c|c|c|c|c|c|}
\hline Fluid & $\begin{array}{c}\text { Density } \\
\rho\left(\mathrm{g} / \mathrm{cm}^{3}\right)\end{array}$ & $\begin{array}{l}\text { Viscosity } \\
\nu\left(\mathrm{cm}^{2} / \mathrm{s}\right)\end{array}$ & $R=5 \mathrm{~cm}$ & $\begin{array}{l}\text { Symbol } \\
R=30 \mathrm{~cm}\end{array}$ & $R=\infty$ \\
\hline Water & 1 & 0.01 & 0 & 0 & $\odot$ \\
\hline $\begin{array}{l}\text { Water }(40 \%) \\
+\operatorname{glycerol}(60 \%)\end{array}$ & 1.16 & 0.086 & $\nabla$ & $\nabla$ & $\nabla$ \\
\hline Silicone V50 & 1 & 0.5 & $\triangleright$ & $\triangleright$ & \\
\hline Silicone V100 & 1 & 1 & & $\triangle$ & \\
\hline Silicone V300 & 1 & 3 & & $\triangleleft$ & \\
\hline Gel & 1.03 & (See Fig. 3) & & $\square$ & \\
\hline
\end{tabular}

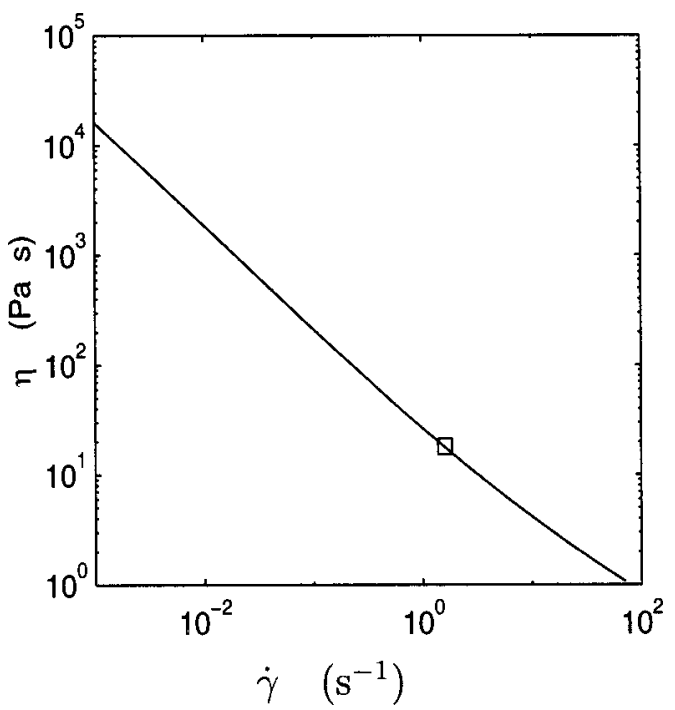

Fig. 3 Dynamic viscosity $\eta$ of the gel versus the shear stress $\dot{\gamma}$. Its density is $\rho=1.03 \mathrm{~g} / \mathrm{cm}^{3}$. We note that this fluid is very viscous in the conditions of our experiments (we can estimate roughly that $\dot{\gamma} \sim 2 \mathrm{U} / \mathrm{d}$ is less than $10 \mathrm{~s}^{-1}$ ). Measurements have been performed with a plane-plane viscosimeter at Ecole des Mines de Paris (CEMEF, Sophia Antipolis).

We consider that the pipe is completely filled when the point $\mathrm{C}$ has reached the point $\mathrm{B}$ (Fig. 2). We define $U_{c}$ as the critical speed beyond which the interface moves until the pipe is completely filled. The speed $v$ is the mean velocity of the point $\mathrm{C}$ moving from A to B (Fig. 2): if $U$ is larger than $U_{c}$, then $v$ is greater than zero.

For the special case of the non-Newtonian fluid gel, the viscosity is smaller where the shear stress is larger, that is near the boundaries. Away from the boundaries, the behavior of the fluid is similar to a solid core pushed by the flow. In other words, the characteristic time of deformation of the interface is very long.

\section{Measurements}

The mean flow velocity $U$ was obtained by measuring the flow rate in the steady-state regime, i.e., when the pipe was completely filled. The flow rate and $v$ were estimated by measuring the filling time of a graded beaker and the travelling time of point $\mathrm{C}$ from $\mathrm{A}$ to B (Fig. 2) with a stopwatch. We may consider that these simple techniques do not lead to any bias error. By repeating the same run (at least five times) for different flow rates, we have estimated that the precision limit and thus the uncertainty was always better than 10 percent on $U$ and $v$.

We measured $v$ as a function of $U$ for different experiments using various fluids, pipes, and curvatures (see Table 1). In Fig. 4, $v$ is plotted versus $U$ for several runs in four different configurations. The line $v=U$ delimits the region where physical solutions exist: $v$ cannot be larger than $U$. In all our experiments $v$ apparently varies linearly with $U$. We may thus determine the critical velocity $U_{c}($ for $v=0)$, the slope $\alpha=\mathrm{d} v / \mathrm{d} U$ and the interfacial velocity $v(U=0)$, by rms fitting a straight line into the data for each fluid, pipe diameter and radius of curvature.

Let us first consider the slope $\alpha$ of the four straight lines in Fig. 4. The analysis presented in the Introduction considered a perfect fluid. It was assumed that the flow is identical in a reference frame moving with the front. In this framework, the filling of a pipe is similar to the emptying of the pipe. This would imply that $\alpha$ is equal to one: adding $\delta U$ to the mean entrance flow velocity would add the same value to the front propagation velocity. It is therefore not surprising that the slopes of the straight lines in Fig. 4 related to water are close to one. When the fluid is far from perfect 


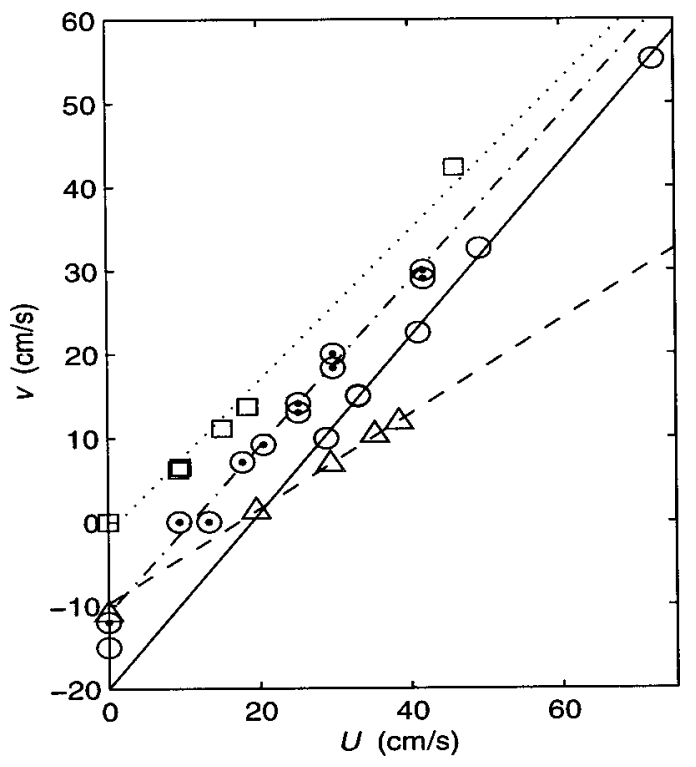

Fig. 4 Speed of the front propagation $v$ versus the mean entrance velocity $U(d=1.5 \mathrm{~cm}$; see list of symbols in Table 1$)$. The straight lines are fitted curves by quadratic means.

but still Newtonian (case of the silicone oil V100 in Fig. 4), $\alpha$ is clearly smaller than one: when the viscosity is large, the deformation of the front strongly depends on $U$. For a low viscosity, the deformation of the interface takes place on a very short time scale and the propagation of the front apparently does not depend on the reference frame. In the other limit, that is for a very large viscosity, the deformation of the front takes place on a very long time scale and the slope is again close to one (this is the case of the non-Newtonian fluid).

The velocity $v(0)$ corresponds to the draining of the pipe. The measurements have been performed by bringing $U$ to zero after filling the pipe: the flow is then from right to left in Fig. 2. It is important to emphasize that $v(0)$ is very small in the case of the non-Newtonian fluid. We have estimated that the draining velocity is about $1 \mathrm{~cm}$ per week. The deformation of the front is visible only when the fluid is sheared and $U_{c}$ is therefore also close to zero for the gel. The agreement in $v(0)$ between the measured values and the values deduced from the fitted straight lines in Fig. 4 is rather good except in the case of water for $R=30 \mathrm{~cm}$. The discrepancy is probably due to measurement errors but also to wetting effects. The contact between the fluid and the PVC is indeed not the same when filling or emptying the pipe. It was not our purpose to investigate wetting effects and we did not test other materials for the pipe. We therefore have to consider that wetting effects may induce a bias error over $U_{c}$ of about 10 percent in the worst case, i.e., an uncertainty of 20 percent in the water case.

The critical speed $U_{c}$ (mean entrance velocity for which $v$ $=0)$ is plotted against the pipe diameter $d$ in Fig. 5. $U_{c}$ clearly tends to increase with $d$. Considering gravitational effects, the larger is $d$, the more efficient are gravity effects to deform the interface. It is thus not surprising that inertial effects have to be increased to compensate for it. Gravity effects could also reasonably depend on the radius of curvature. The interface deforms more rapidly when the pipe is curved: for a same diameter, $U_{c}$ is therefore smaller when $R=\infty$. Otherwise, if $R$ is large but not infinite, the travelling time of the front in the pipe is large and the mean entrance velocity has to be large for the front to propagate. In other words, for a very small radius, the front has not enough time to deform before the exit of the pipe. This would explain the fact that $U_{c}$ is larger for $R=30 \mathrm{~cm}$ than for $R=5 \mathrm{~cm}$.

In addition, above $\nu=1 \mathrm{~cm}^{2} / \mathrm{s}$, viscous effects tend to maintain the shape of the interface and thus decrease $U_{c}$. This is noticeable

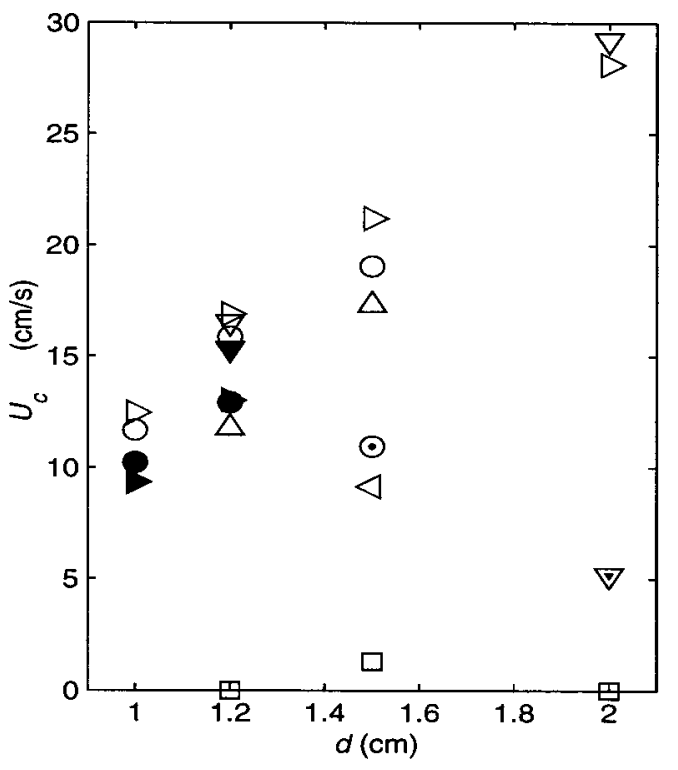

Fig. 5 Critical speed versus the pipe diameter (see list of symbols in Table 1)

for the silicone V100 and clearly visible for the silicone V300. Again the case of the gel requires specific comments. $U_{c}$ is very close to zero because the front deforms only when the fluid is sheared as mentioned above. The graphical estimation of $U_{c}$ gives a nonzero value only for $d=1.5 \mathrm{~cm}$. This configuration will allow us to estimate dimensionless numbers for the gel in the next section. 2 .

The data in Figs 4 and 5 are available in tabular form in Table

\section{Interpretation in Terms of Dimensionless Numbers}

We may consider various dimensionless numbers that could characterize the flow in our special configuration. Note that in our experiments both the Bond number $\left(\mathrm{Bo}=d^{2} \rho g / \sigma\right)$ and the Weber number $\left(\mathrm{We}=\rho d U^{2} / \sigma\right)$ are very large: capillary effects are a lot weaker than inertial and gravitational effects.

Considering inertial and viscous effects in the framework of a curved pipe, we introduce a modified Reynolds number Re, which partly takes into account centrifugal effects, and is in fact the classical Reynolds number plus the Dean number [15]:

$$
\mathrm{Re}=\frac{U d}{\nu}\left(1+\sqrt{\frac{d}{R}}\right) .
$$

To balance inertial and gravity effects, we build a modified Froude number Fr, such as:

$$
\mathrm{Fr}=\frac{U}{\sqrt{g d}}\left(1+\sqrt{\frac{d}{R}}\right) .
$$

The modification of the classical dimensionless numbers may be understood by considering that there is a need to add centrifugal effects to maintain the fluid against the concave side of the pipe. Note that (2) and (3) tend toward the classical Reynolds and Froude numbers in the limiting case of a straight pipe, i.e., when $R$ tends toward infinity.

Let us finally define $K$, which measures the balance between gravity and viscosity:

$$
K=\frac{\mathrm{Fr}}{\operatorname{Re}}=\frac{\nu}{\sqrt{g d^{3}}} .
$$


Table 2 Measured values

\begin{tabular}{|c|c|c|c|c|c|}
\hline & Fluid & $\begin{array}{c}\text { Radius } \\
\text { of curvature } \\
R(\mathrm{~cm})\end{array}$ & $\begin{array}{c}\text { Pipe } \\
\text { diameter } \\
d(\mathrm{~cm})\end{array}$ & $\begin{array}{c}\text { Speed } \\
\text { of front } \\
v(\mathrm{~cm} / \mathrm{s})\end{array}$ & $\begin{array}{c}\text { Mean entrance } \\
\text { velocity } \\
U(\mathrm{~cm} / \mathrm{s})\end{array}$ \\
\hline \multicolumn{2}{|c|}{ Silicone V50 } & 5 & 1.0 & -8.6 & 0.0 \\
\hline & - & 5 & 1.0 & 0.6 & 10.2 \\
\hline & - & 5 & 1.0 & 3.2 & 14.1 \\
\hline & - & 5 & 1.0 & 11.8 & 28.7 \\
\hline & - & 5 & 1.0 & 18.9 & 36.6 \\
\hline & - & 5 & 1.2 & -10.7 & 0.0 \\
\hline & - & 5 & 1.2 & 4.4 & 19.8 \\
\hline & - & 5 & 1.2 & 6.7 & 22.8 \\
\hline & - & 5 & 1.2 & 12.2 & 31.4 \\
\hline \multirow[t]{22}{*}{$\triangleright$} & - & 30 & 1.0 & -8.8 & 0.0 \\
\hline & - & 30 & 1.0 & 1.0 & 13.0 \\
\hline & - & 30 & 1.0 & 4.9 & 18.9 \\
\hline & - & 30 & 1.0 & 8.9 & 23.6 \\
\hline & - & 30 & 1.0 & 19.5 & 35.6 \\
\hline & - & 30 & 1.0 & 22.1 & 38.4 \\
\hline & - & 30 & 1.2 & -10.8 & 0.0 \\
\hline & - & 30 & 1.2 & 1.4 & 19.1 \\
\hline & - & 30 & 1.2 & 4.5 & 24.0 \\
\hline & - & 30 & 1.2 & 8.9 & 31.0 \\
\hline & - & 30 & 1.2 & 16.9 & 43.6 \\
\hline & - & 30 & 1.5 & -13.6 & 0.0 \\
\hline & - & 30 & 1.5 & 2.2 & 24.2 \\
\hline & - & 30 & 1.5 & 7.3 & 34.2 \\
\hline & - & 30 & 1.5 & 16.4 & 49.2 \\
\hline & - & 30 & 1.5 & 22.5 & 57.0 \\
\hline & - & 30 & 2.0 & -18.0 & 0.0 \\
\hline & - & 30 & 2.0 & 2.0 & 34.6 \\
\hline & - & 30 & 2.0 & 3.4 & 38.4 \\
\hline & - & 30 & 2.0 & 4.8 & 41.6 \\
\hline & - & 30 & 2.0 & 6.4 & 46.3 \\
\hline & - & 30 & 2.0 & 11.2 & 62.4 \\
\hline \multicolumn{2}{|c|}{$\Delta$ silcone V100 } & 30 & 1.2 & -8.5 & 0.0 \\
\hline & - & 30 & 1.2 & 2.3 & 14.7 \\
\hline & - & 30 & 1.2 & 2.3 & 14.7 \\
\hline & - & 30 & 1.2 & 3.9 & 17.1 \\
\hline & - & 30 & 1.2 & 5.0 & 18.0 \\
\hline & - & 30 & 1.5 & -11.0 & 0.0 \\
\hline & - & 30 & 1.5 & 1.3 & 19.6 \\
\hline & - & 30 & 1.5 & 6.8 & 29.4 \\
\hline & - & 30 & 1.5 & 10.3 & 35.3 \\
\hline & - & 30 & 1.5 & 11.8 & 38.5 \\
\hline \multicolumn{2}{|c|}{$\triangleleft$ Silicone V300 } & 30 & 1.5 & -6.2 & 0.0 \\
\hline & - & 30 & 1.5 & 0.9 & 10.5 \\
\hline \multicolumn{2}{|c|}{ water } & 5 & 1.0 & -9.5 & 0.0 \\
\hline & - & 5 & 1.0 & 3.0 & 12.4 \\
\hline & - & 5 & 1.0 & 7.2 & 15.5 \\
\hline & - & 5 & 1.0 & 10.8 & 18.1 \\
\hline & - & 5 & 1.2 & -10.3 & 0.0 \\
\hline & - & 5 & 1.2 & 0.7 & 13.7 \\
\hline & - & 5 & 1.2 & 1.4 & 14.0 \\
\hline & - & 5 & 1.2 & 7.0 & 19.1 \\
\hline & - & 5 & 1.5 & -11.9 & 0.0 \\
\hline \multirow[t]{21}{*}{$\mathrm{O}$} & - & 30 & 1.0 & -9.8 & 0.0 \\
\hline & - & 30 & 1.0 & 3.2 & 14.8 \\
\hline & - & 30 & 1.0 & 9.6 & 19.4 \\
\hline & - & 30 & 1.0 & 13.5 & 23.1 \\
\hline & - & 30 & 1.0 & 41.3 & 47.1 \\
\hline & - & 30 & 1.2 & -12.0 & 0.0 \\
\hline & - & 0 & 1.2 & 0.5 & 16.9 \\
\hline & - & 30 & 1.2 & 4.6 & 19.5 \\
\hline & - & 30 & 1.2 & 5.6 & 22.5 \\
\hline & - & 30 & 1.2 & 9.2 & 23.2 \\
\hline & - & 30 & 1.2 & 14.1 & 29.0 \\
\hline & - & 30 & 1.2 & 28.0 & 42.3 \\
\hline & - & 30 & 1.2 & 28.8 & 44.9 \\
\hline & - & 30 & 1.2 & 52.7 & 66.6 \\
\hline & - & 30 & 1.5 & -15.0 & 0.0 \\
\hline & - & 30 & 1.5 & 9.9 & 28.9 \\
\hline & - & 30 & 1.5 & 15.0 & 33.1 \\
\hline & - & 30 & 1.5 & 14.9 & 33.1 \\
\hline & - & 30 & 1.5 & 22.5 & 41.1 \\
\hline & - & 30 & 1.5 & 32.6 & 49.2 \\
\hline & - & 30 & 1.5 & 55.2 & 72.2 \\
\hline$\odot$ & - & $\infty$ & 1.5 & -12.0 & 0.0 \\
\hline & - & $\infty$ & 1.5 & 0.0 & 9.4 \\
\hline & - & $\infty$ & 1.5 & 0.0 & 13.3 \\
\hline & - & $\infty$ & 1.5 & 7.1 & 17.8 \\
\hline & - & $\infty$ & 1.5 & 9.2 & 20.6 \\
\hline & - & $\infty$ & 1.5 & 13.0 & 25.2 \\
\hline
\end{tabular}

Table 2 (Continued)

\begin{tabular}{|c|c|c|c|c|}
\hline Fluid & $\begin{array}{c}\text { Radius } \\
\text { of curvature } \\
R(\mathrm{~cm})\end{array}$ & $\begin{array}{c}\text { Pipe } \\
\text { diameter } \\
d(\mathrm{~cm})\end{array}$ & $\begin{array}{c}\text { Speed } \\
\text { of front } \\
v(\mathrm{~cm} / \mathrm{s})\end{array}$ & $\begin{array}{c}\text { Mean entrance } \\
\text { velocity } \\
U(\mathrm{~cm} / \mathrm{s})\end{array}$ \\
\hline- & $\infty$ & 1.5 & 14.1 & 25.2 \\
\hline - & $\infty$ & 1.5 & 18.3 & 29.8 \\
\hline- & $\infty$ & 1.5 & 20.0 & 29.8 \\
\hline - & $\infty$ & 1.5 & 29.0 & 41.8 \\
\hline- & $\infty$ & 1.5 & 30.0 & 41.8 \\
\hline $\begin{array}{c}\nabla \text { water }(40 \%) \\
+ \text { glyc. }(60 \%)\end{array}$ & 5 & 1.2 & 1.6 & 16.7 \\
\hline $\begin{array}{c}8+(00) \\
-\end{array}$ & 5 & 1.2 & 4.8 & 20.2 \\
\hline- & 5 & 1.2 & 12.6 & 27.5 \\
\hline$\nabla$ & 30 & 1.2 & 6.1 & 26.7 \\
\hline- & 30 & 1.2 & 15.0 & 38.8 \\
\hline- & 30 & 1.2 & 14.9 & 38.8 \\
\hline- & 30 & 1.2 & 29.5 & 63.2 \\
\hline- & 30 & 1.2 & 51.6 & 95.7 \\
\hline & 30 & 2.0 & 5.7 & 39.3 \\
\hline- & 30 & 2.0 & 3.3 & 42.4 \\
\hline - & 30 & 2.0 & 10.2 & 56.8 \\
\hline - & 30 & 2.0 & 15.2 & 64.2 \\
\hline - & $\infty$ & 2.0 & 0.0 & 6.9 \\
\hline$\nabla$ & $\infty$ & 2.0 & 0.0 & 8.2 \\
\hline - & $\infty$ & 2.0 & 16.4 & 24.8 \\
\hline - & $\infty$ & 2.0 & 22.7 & 32.1 \\
\hline - & $\infty$ & 2.0 & 34.9 & 47.1 \\
\hline - & $\infty$ & 2.0 & 45.3 & 69.2 \\
\hline- & $\infty$ & 2.0 & 57.0 & 80.4 \\
\hline$\square$ gel & 30 & 1.2 & 0.0 & 0.0 \\
\hline$-\infty \quad$ & 30 & 1.2 & 1.8 & 2.0 \\
\hline - & 30 & 1.2 & 20.3 & 23.1 \\
\hline - & 30 & 1.2 & 22.3 & 27.8 \\
\hline - & 30 & 1.5 & 0.0 & 0.0 \\
\hline - & 30 & 1.5 & 6.2 & 9.3 \\
\hline - & 30 & 1.5 & 6.5 & 9.6 \\
\hline - & 30 & 1.5 & 11.1 & 15.1 \\
\hline - & 30 & 1.5 & 13.7 & 18.5 \\
\hline - & 30 & 1.5 & 42.3 & 46.0 \\
\hline- & 30 & 2.0 & 0.0 & 0.0 \\
\hline - & 30 & 2.0 & 38.7 & 49.6 \\
\hline- & 30 & 2.0 & 35.9 & 53.6 \\
\hline - & 30 & 2.0 & 31.0 & 55.6 \\
\hline- & 30 & 2.0 & 44.1 & 64.0 \\
\hline - & 30 & 2.0 & 55.9 & 88.9 \\
\hline
\end{tabular}

Note that $K$ does not depend on the mean flow velocity $U$. Re and Fr are computed for $U=U_{c}$ and plotted against $K$ in Figs. 6 and 7.

We may consider two kinds of estimation of the dimensionless numbers for the gel. First we may use the graphical estimation of $U_{c}$ from Fig. 4 for $d=1.5 \mathrm{~cm}: U_{c} \sim 1.2 \mathrm{~cm} / \mathrm{s}$, leading to $\nu \sim 200$ $\mathrm{cm}^{2} / \mathrm{s}$ from Fig. 3 (assuming that $\dot{\gamma} \sim 2 U_{c} / d$ ). This allows us to plot the squares in Figs. 6 and 7. Second, we may estimate from our measurement that $U_{c} \sim v(0)$ is nonzero but very small (six orders of magnitude less than the other values of velocity). In that case, the squares would be far off the bottom right-hand corners of Figs. 6 and 7. In any case, the gel is possibly following the trend of Newtonian fluids with very large viscosity.

In Fig. 6, the data can be separated into two regions corresponding to partially (below the data) and completely (above the data) filled pipes. In a situation where the flow is characterized by a point belonging to the border line between the two regions, the filling would be effective over an infinite time, using an infinite amount of fluid. In the frame of a linear approximation, the data follow approximatively the $(-1)$ slope, showing that $\operatorname{Fr}(U$ $=U_{c}$ ) is constant from Eq. (4)

Another way to represent the same results is given in Fig. 7. One sees that $\mathrm{Fr}$ is of the same order of magnitude for all experiments while $K$ varies over four orders of magnitude, but Fr is not rigorously constant. In the range of diameters we investigated, we can say roughly that Fr characterizes the ability to fill the pipes 


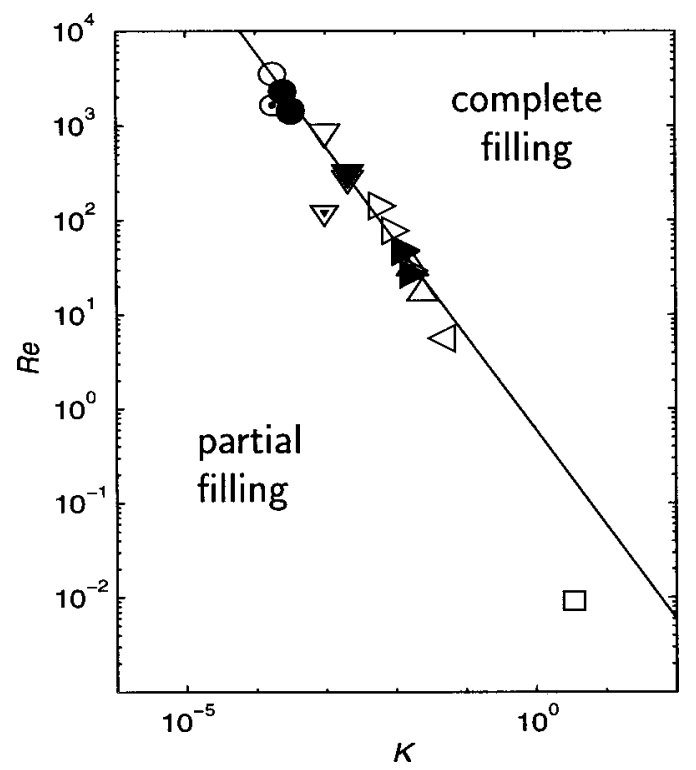

Fig. 6 Modified Reynolds number (2) computed for $U=U_{c}$ versus $K(4)$ (see list of symbols in Table 1.)

( $F r=1$ corresponds to the theoretical limit (1) where the flow detaches tangentially at the exit of a horizontal box, as suggested above in the introduction). Setting a flow rate leading to $\mathrm{Fr} \geqslant 1$ would certainly fill any straight pipe.

A careful examination of Fig. 7 leads to several remarks. For Newtonian fluids, the dotted symbols, which correspond to straight pipes, lie below the points corresponding to curved pipes. The latter are gathered with no obvious dependence on the radius of curvature $R$. Therefore the modified Froude number, which takes into account centrifugal effects, can predict the effective filling of the curved pipes, but is of less interest in the limit of straight pipes. Each group of points corresponds to a different fluid, i.e., viscosity. The tendency for $\operatorname{Fr}\left(U=U_{c}\right)$ to increase with $d$ is clearly visible in each group of points. For $K \geqslant 10^{-2}, \operatorname{Fr}(U$ $=U_{c}$ ) is apparently decreasing with $K$ and the flow would eventually tend to follow a non Newtonian behavior.

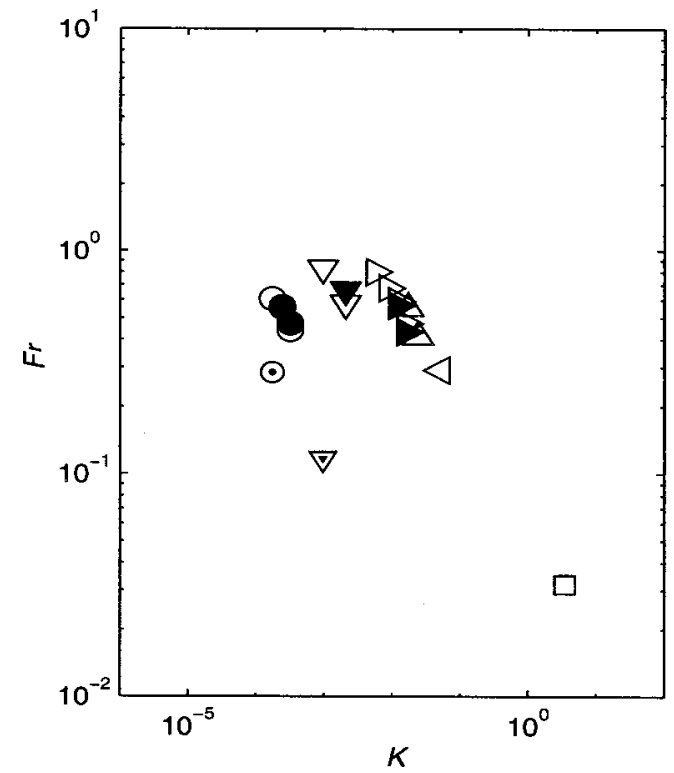

Fig. 7 Modified Froude number (3) computed for $\boldsymbol{U}=\boldsymbol{U}_{c}$ versus $K(4)$ ( see list of symbols in Table 1.)
In almost all our experiments, Re is larger than 10 (see Fig. 6) indicating that viscous effects are smaller than inertial ones. Meanwhile, the data on Fr indicate that both inertial and gravitational effects are comparable.

\section{Conclusion}

In order to understand the failure in filling curved pipes, we performed a series of experiments using various fluids. We measured the velocity of propagation of the front $v$ and we deduced from its linear dependence with the mean flow velocity $U$ a critical value $U_{c}$, below which there is no longer a complete filling of the pipe. This critical velocity $U_{c}$ is increasing with $d$ for each fluid.

Results are presented in a synthetic way as relations between dimensionless numbers. We have shown, in the scope of our work, that a modified Froude number determines the ability of the flow to fill a curved pipe. In all our experiments and for Newtonian fluids of different viscosities, the filling was complete for $\mathrm{Fr}$ $\geqslant 0.6 \pm 0.2$.

We also performed experiments with a non-Newtonian fluid whose rheological behavior is similar to the one of cement suspensions. The front deforms very slowly when the shear is weak because this increases the viscosity of the fluid. In that case, the complete filling may be obtained for Fr very small. Following $[16,17]$, a more extensive study would be needed in order to understand the detail of the non-Newtonian effects.

For industrial applications we advise considering pipes of small diameters and injecting the solution at a relatively low flow rate in order to increase its effective viscosity. However, a future study of interest will be to repeat the present experiments in the context of slug flows.

\section{Acknowledgments}

The authors thank C. Peiti at Ecole des Mines de Paris (CEMEF, Sophia Antipolis) for his help, and R. Leroy and O. Coussy for providing helpful comments. The authors are grateful to J.Ch. Bery for his help in the conception and realization of the experimental apparatus. This study was part of a contract with the Laboratoire Central des Ponts et Chaussées.

\section{Nomenclature}

$$
\begin{aligned}
d & =\text { diameter of pipe } \\
R & =\text { radius of curvature } \\
g & =\text { acceleration of gravity } \\
\nu & =\text { kinematic viscosity } \\
\rho & =\text { density } \\
U & =\text { mean entrance velocity } \\
u & =\text { mean exit velocity } \\
v & =\text { velocity of the upper point of } \\
& \text { the front } \\
h & =\text { height of the exit flow } \\
U_{c}=U(v=0) & \text { critical mean velocity } \\
\operatorname{Fr}=U\left(1+(d / R)^{1 / 2}\right) /(g d)^{1 / 2} & =\text { modified Froude number } \\
\operatorname{Re}=U d\left(1+(d / R)^{1 / 2}\right) / \nu & =\text { modified Reynolds number } \\
K & =\text { Fr/Re } \\
\sigma & =\text { surface tension } \\
L_{c}=\sigma^{1 / 2}(\rho g)^{-1 / 2} & =\text { capillary length } \\
\mathrm{Bo}=d^{2} \rho g / \sigma & =\text { Bond number } \\
\mathrm{We}=\rho d U^{2} / \sigma & =\text { Weber number }
\end{aligned}
$$

\section{References}

[1] Berger, S. A., Talbot, L., and Yao, L.-S., 1983, "Flow in curved pipes," Annu. Rev. Fluid Mech., 15, pp. 461-512.

[2] Soh, W. Y., and Berger, S. A., 1984, "Laminar entrance flow in a curved pipe," J. Fluid Mech., 148, pp. 109-135.

[3] Olson, D. E., and Snyder, B., 1985, "'The upstream scale of flow development in curved circular pipes," J. Fluid Mech., 150, pp. 139-158.

[4] Ishigaki, H., 1994, "Analogy between laminar flows in curved pipes and orthogonally rotating pipes,'” J. Fluid Mech., 268, pp. 133-145. 
[5] Popiel, C. O., and van der Merwe, D. F., 1996, "Friction factor in sine-pipe flow," ASME J. Fluids Eng., 118, pp. 341-345.

[6] Majumdar, B., Mohan, R., Singh, S. M., and Agrawal, D. P., 1998, “'Experimental study of flow in a high aspect ratio 90 deg curved diffuser," ASME J. Fluids Eng., 120, pp. 83-89.

[7] Chanson, H., 1997 "Air-water flows in partially-filled conduits," J. Hydraul. Res. 35, No. 5, pp. 591-602.

[8] Capart, H., Sillen, X., and Zech, Y., 1997, “The design of sewage sludge pumping systems,', J. Hydraul. Res., 35, No. 5, pp. 659-672.

[9] Woods, B. D., and Hanratty, T. J., 1999, "Influence of Froude number on physical processes determining frequency of slugging in horizontal gas-liquid flows,', Int. J. Multiphase Flow, 25, No. 6-7, pp. 1195-1223.

[10] Taitel, Y., Sarica, C., and Brill, J. P., 2000, "Slug flow modeling for downward inclined pipe flow: theoretical considerations,' Int. J. Multiphase Flow, 26, No. 5, pp. 833-844.
[11] Benjamin, T. B., 1968, “Gravity currents and related phenomena,' J. Fluid Mech., 31, pp. 209-248.

[12] Simpson, J. E., 1982, "Gravity currents in the laboratory, atmosphere, and ocean,' Annu. Rev. Fluid Mech., 14, pp. 213-234.

[13] Asavenant, J., and Vanden-Broeck, J.-M., 1996, "Nonlinear free-surface flows emerging from vessels and flows under a sluice gate," J. Austral. Math. Soc., B 38, pp. 63-86.

[14] Vanden-Broeck, J.-M., and Keller, J. B., 1987, “Weir flows,” J. Fluid Mech., 176, pp. 283-293.

[15] Dean, W. R., 1927, "Note on the motion of fluid in a curved pipe," Philos. Mag., 20, pp. 208-223.

[16] Chilton, R., Stainsby, R., and Thompson, S., 1996, "The design of sewage sludge pumping systems,", J. Hydraul. Res., 34, No. 3, pp. 395-408.

[17] Chilton, R., and Stainsby, R., 1998, "Pressure loss equations for laminar and turbulent non-Newtonian pipe flow,', J. Hydraul. Eng., 124, No. 5, pp. 522529. 